\title{
1 Reply to: Nutrient scarcity cannot cause mast seeding
}

3 Authors: M. Fernández-Martínez ${ }^{* 1}$, J. Sardans ${ }^{2,3}$, F. Sayol ${ }^{4,5}$, J.M. LaMontagne ${ }^{6}$, M.

4 Bogdziewicz ${ }^{7}$, A. Collalti ${ }^{8,9}$, A. Hacket-Pain ${ }^{10}$, G. Vacchiano ${ }^{11}$, J.M. Espelta ${ }^{3}$, J. Peñuelas ${ }^{2,3}$, I.A.

5 Janssens ${ }^{1}$

6 Affiliations:

71 Research Group PLECO (Plants and Ecosystems), Department of Biology, University of 8 Antwerp, 2610 Wilrijk, Belgium.

$9 \quad{ }^{2}$ CSIC, Global Ecology Unit, CREAF-CSIC-UAB, Bellaterra 08193 Barcelona, Catalonia, Spain

$10{ }^{3}$ CREAF, Cerdanyola del Vallès, 08193 Barcelona, Catalonia, Spain

$11{ }^{4}$ Department of Biological and Environmental Sciences, University of Gothenburg, SE 40530

12 Gothenburg, Sweden

$13{ }^{5}$ Gothenburg Global Biodiversity Centre, SE 40530 Gothenburg, Sweden

$14{ }^{6}$ Department of Biological Sciences, DePaul University, Chicago, IL, USA

157 Department of Systematic Zoology, Faculty of Biology, Adam Mickiewicz University in Poznań, 16 Poland

$17{ }^{8}$ Institute for Agriculture and Forestry Systems in the Mediterranean, National Research Council 18 of Italy (CNR-ISAFOM), 87036, Rende (CS), Italy

199 Department of Innovation in Biological, Agro-food and Forest Systems, University of Tuscia, 2001100 Viterbo, Italy

2110 Department of Geography and Planning, School of Environmental Sciences, University of 22 Liverpool, Liverpool, UK

2311 Università di Milano, DISAA. Via Celoria 2, 20133, Milan - Italy

24 * Correspondence to: M. Fernández-Martínez, marcos.fernandez-martinez@uantwerpen.be

25 Length of the main text: 1201 words

26 Figures: 2 figures

27 References: 15 
29 Kelly's commentary regarding our paper ${ }^{1}$, claiming that nutrient scarcity cannot select 30 for masting behaviour in plants, initiated a fruitful discussion on traditionally settled 31 hypotheses about the evolution of reproductive behaviour in plants. In his commentary, 32 Prof. Kelly raises support for a contrasting hypothesis explaining our observation that temporally variable seed production is more pronounced under nutrient scarcity, namely that nutrient scarcity does not directly cause seed production variability, but instead increases variability induced by economies of scale. The commentary hinges mainly on the argument that an economy of scale (EOS) is necessary to select for highly variable seed production. It also points out that there are no mechanisms by which nutrient scarcity would select for that particular trait over generations. In reply to the stimulating comment, we 1) propose a mechanism by which nutrient scarcity may select for highly variable seed production, with weather patterns inducing masting synchrony across populations; 2) further discuss why wind-pollination and predator satiation, the EOS suggested by Kelly, cannot be the only selective pressures that select for highly variable reproduction.

44 (1) There is robust empirical evidence ${ }^{2,3}$ showing that nutrient scarcity, and climate, are long existing evolutionary forces that have selected for multiple plant traits and have constrained the physiology of plants since their early development. Limiting resources, such as water and nutrients, thus trigger the evolution of conservative traits for those limiting factors ${ }^{3}$. Logically, nutrient availability is a direct determinant of the mean fruit production in agriculture and in the wild ${ }^{4}$. In our paper ${ }^{1}$, we hypothesised that low nutrient availability is also an important factor selecting for highly variable and synchronized seed production, the latter in combination with adaptation to variability in

52 long-term climate patterns. Our hypothesis as to why nutrient scarcity may have selected for highly variable seed production in nutrient-poor plants, probably not entirely explained in our original paper, was based on a mechanism linking highly 
55 variable seed production in nutrient-poor plant species to increased inter- and 56 intraspecific competitiveness.

57 Because fruits are nutrient-enriched tissues ${ }^{5}$, their production under low fertility implies 58 a reduced allocation of nutrients to growth and defence ${ }^{6}$, and therefore lower 59 competitiveness and survival for the parent plants. Reductions in plant nutrient concentrations after reproduction have been described for several species ${ }^{7}$, in addition to growth and defence-reproductive trade-offs ${ }^{6}$. Therefore, when nutrients are scarce, losing large amounts of nutrients year after year might jeopardize plant growth through reduced photosynthesis, a highly nutrient-dependent process ${ }^{8}$. A constant yearly reproduction would also imply a constant lowering of the availability of nutrients for other processes. In contrast, nutrient accumulation in years with suitable weather conditions for soil organic matter decomposition and mineralization may provide sufficient nutrients to allow a high fruit crop in the following year, which would not come at the expense of reduced competitiveness or increased mortality risk (Figure 1). Under these conditions, high temporal variability would thus be beneficial and likely to be selected for. In contrast, under nutrient-rich conditions, plants can potentially reproduce regularly without jeopardising their competitiveness; this is actually one of the reasons for fertilizer addition as a long-existing agricultural practice. This mechanism, which could have originated during the early evolution of plants, may explain why, under low nutrient availability, nutrient-conservative plants with highly variable reproduction may have been preferentially selected in comparison to nutrientspending plants (with more constant reproduction). Further research including longterm data sets of reproduction, growth and defence allocation, however, is still needed to validate our hypotheses.

For a population to exhibit highly variable reproduction over time, a strong synchrony among individuals is required ${ }^{9}$. Synchronous seed production is another important feature of masting behaviour that has been traditionally associated with the benefits of 
82 EOS, as it has been suggested to be an adaptive response to improve pollination 83 efficiency or escape seed predation ${ }^{7}$. Synchrony among individuals in a plant 84 population is the rule rather than the exception, as for example in leaf flushing, flower blooming, die-back episodes or simply growth as shown by dendrochronology studies. The most likely mechanism driving the synchrony in phenology, growth or reproduction is the similar response of a population to changing weather patterns, by affecting metabolism and plant resources.

Over time, the scientific community has discounted environmental effects based in part on two assumptions that may not hold true, and this has fed the line of thought that EOS benefits are necessary for high variable seed production to evolve. First, a direct role of weather in synchronizing seed production has been discounted in part because interannual variation in weather is much less than variation in seed production. We argue that this line of argument does not hold because the link between weather and seed production in a given year may be highly non-linear ${ }^{10}$. In Mediterranean regions, for example, wet spring weather may simultaneously increase both carbon availability through photosynthesis and nutrient availability through mineralization ${ }^{11}$, boosting plant resources and seed production. Second, proportional allocation has been assumed to describe the passive allocation of resources to reproduction ${ }^{7}$. However, work in the fields of ecological stoichiometry and carbon dynamics suggest that plants allocate resources based on a hierarchy of needs, in which a set portion of resources goes toward tissue maintenance at the expense of all other functions ${ }^{4}$. We, therefore, suggest that interannual variability in reproduction is more likely to have been evolutionary selected than synchrony.

(2) From evolutionary and theoretical bases, that highly variable reproduction can only be selected because of wind pollination and predator satiation does not hold either. For that to be true, we would have to accept that the common ancestors of plants, before the trait of wind pollination and their seed predators evolved, did not show variable 
seed production. In fact, it implies that the reproductive efforts of organisms other than

110 vascular plants, which do not produce pollen or may not have important predators of

111 their offspring, cannot be temporally variable unless, for other reasons, it evolved later

112 in these clades. However, there is evidence suggesting that other organisms that reproduce sexually are also temporally synchronised and variable (Figure 2). Some bryophytes tend to produce sporophytes more frequently than others ${ }^{12}$, and their production is mainly controlled by weather variability, like in vascular plants ${ }^{13}$, being synchronised in time and space. The same happens with mushroom communities, being highly synchronised, interannually variable, and highly dependent on weather conditions ${ }^{14}$. Even the rates of animal fertility vary amongst years depending on the amount of resources available, such as fertility rates of wild boars ${ }^{15}$. Given the fact that temporally variable sexual reproduction in nature seems to be common, we should conclude that factors others than wind pollination and predator satiation may have played a role in shaping this reproductive trait. We suggest that one of these potential factors triggering a highly variable seed production, before wind pollination and predators evolved, may have been nutrient scarcity because of its role in determining the physiology of a broad range of organisms ${ }^{3}$. Later on, wind pollination and predator satiation may have evolved, reinforcing a highly variable seed production of vascular plants. In order to better understand the evolutionary history of highly variable reproduction in vascular plants, the field of masting will benefit from broadening its scope and looking for inspiration in different disciplines and exploring examples from other groups of organisms.

\section{References:}

132 1. Fernández-Martínez, M. et al. Nutrient scarcity as a selective pressure for mast

2. Fernández-Martínez, M. et al. Sea spray influences water chemical composition of Mediterranean semi-natural springs. Catena 173, 414-423 (2019).

3. Peñuelas, J. et al. The bioelements, the elementome, and the biogeochemical 
niche. Ecology 100, e02652 (2019).

4. Fernández-Martínez, M., Vicca, S., Janssens, I. A., Espelta, J. M. \& Peñuelas, J. The role of nutrients, productivity and climate in determining tree fruit production in European forests. New Phytol. 213, 669-679 (2016).

5. Reekie, E. G. \& Bazzaz, F. A. Reproductive effort in plants. II Does carbon reflect the allocation of other resources? Am. Nat. 129, 897-906 (1987).

6. Redmond, M. D., Davis, T. S., Ferrenberg, S. M. \& Wion, A. P. Resource allocation trade-offs in a mast-seeding conifer: Piñon pine prioritizes reproduction over defense. AoB Plants 11, 1-11 (2019).

7. Pearse, I. S., Koenig, W. D. \& Kelly, D. Mechanisms of mast seeding: resources, weather, cues, and selection. New Phytol. 212, 546-562 (2016).

8. Wright, I. J. et al. The worldwide leaf economics spectrum. Nature 428, 821-7 (2004).

9. Cardinale, B. J. et al. Biodiversity loss and its impact on humanity. Nature 489, 326-326 (2012).

10. Fernández-Martínez, M., Bogdziewicz, M., Espelta, J. M. \& Peñuelas, J. Nature beyond Linearity: Meteorological Variability and Jensen's Inequality Can Explain Mast Seeding Behavior. Front. Ecol. Evol. 5, 1-8 (2017).

11. Fernández-Martínez, M., Garbulsky, M., Peñuelas, J., Peguero, G. \& Espelta, J. M. Temporal trends in the enhanced vegetation index and spring weather predict seed production in Mediterranean oaks. Plant Ecol. 216, 1061-1072 (2015).

12. Fernández-Martínez, M. et al. Monitoring Spatial and Temporal Variabilities of Gross Primary Production Using MAIAC MODIS Data. Remote Sens. 11, 874 (2019).

13. Rydgren, K. \& Økland, R. H. Sex distribution and sporophyte frequency in a population of the clonal moss Hylocomium splendens. J. Bryol. 24, 207-214 (2002).

14. Martínez de Aragón, J., Riera, P., Giergiczny, M. \& Colinas, C. Value of wild mushroom picking as an environmental service. For. Policy Econ. 13, 419-424 (2011).

15. Groot Bruinderink, G. W. T. A., Hazebroek, E. \& Van Der Voot, H. Diet and condition of wild boar, Sus scrofu scrofu, without supplementary feeding. J. Zool. 
170

171 Correspondence and requests for materials should be addressed to:

172

Marcos Fernández-Martínez: marcos.fernandez-martinez@uantwerpen.be

173

174 Conflict of interest

175 The authors declare no conflict of interest.

176

177 Author contribution

178 M.F-M., J.S., J.P., J.M.E., J.P. and I.A.J., conceived the paper. All authors contributed 179 equally to the writing of the manuscript.

180 
182 Figure 1: Scheme showing the hypothesised mechanism by which nutrient scarcity may act as a selective pressure of variable reproduction. When nutrients are scarce, delaying reproductive efforts may provide a competitive advantage given by

185 the lower rate of nutrient loss through reproductive structures. Losing nutrients through 186 reproduction may reduce growth in the short term by decreasing photosynthetic rates. 187 Under low nutrient availability, nutrient-conservative plants (A, with more variable reproduction) outcompete, by vegetative growth, nutrient-spending plants ( $B$, less variable reproduction). This competition may thus increase the probability of seed germination in nutrient-conservative plants and therefore potentially select for nutrientconservative traits such as high interannually variable reproduction. Synchronous reproduction would then be the logical response to reproducing only when weather is optimal and also selected for during evolution.
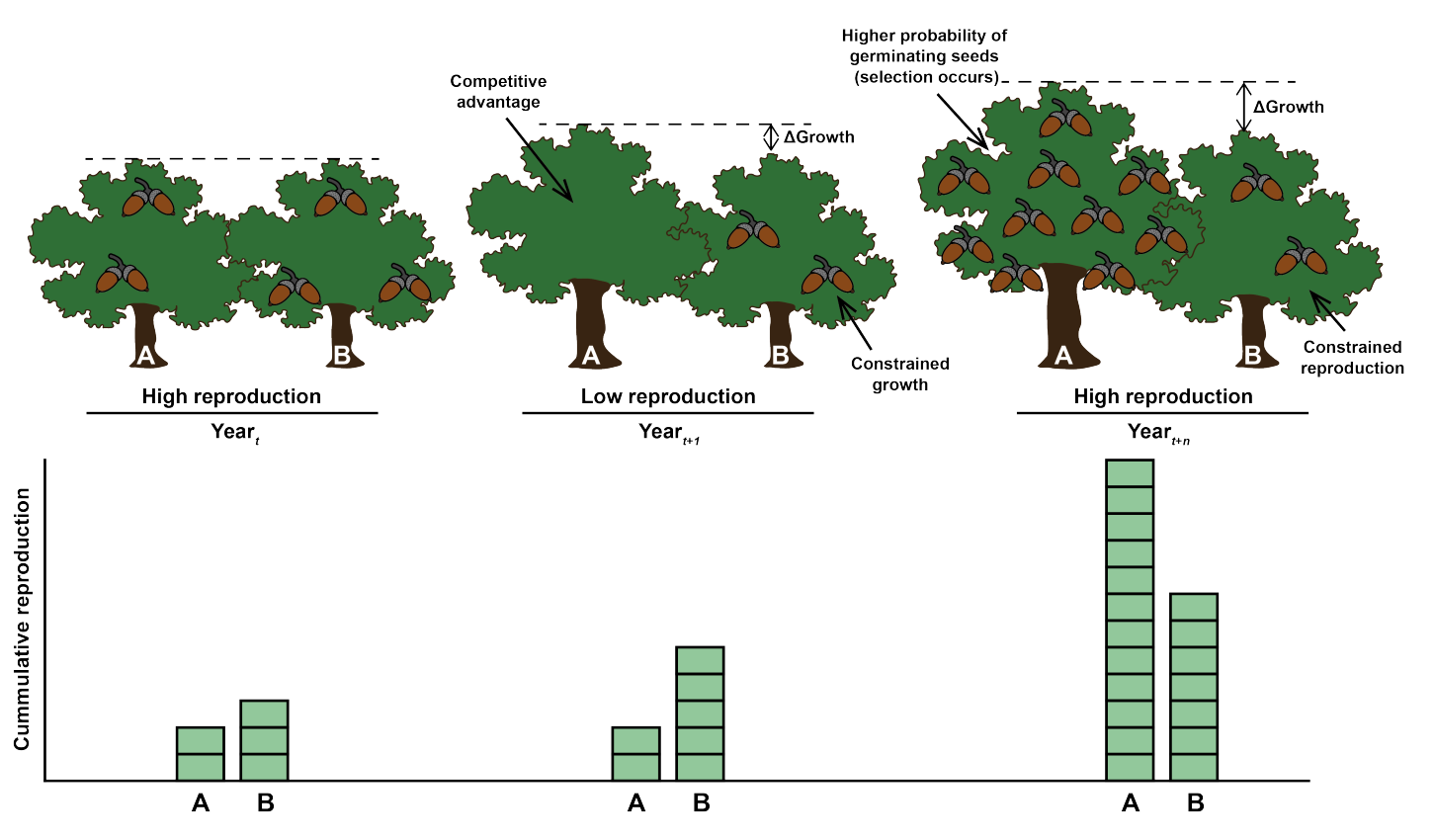
197 Sporophytes of Plagiomnium undulatum (a species that produces sporophytes only 198 rarely); (b) acorn production by a Quercus ilex tree (often considered a masting species); (c) mushroom production by Hypholoma fasciculare (fungal reproduction is highly interannually synchronised within and across species); (d) a mother wild boar with four piglets (sow fertility rates depend on the availability of food, such as acorns, during Quercus mast years). The photographs in (a) and (b) were taken by Catherine Preece, the photograph in (c) was taken by Jordi Corbera, and the photograph in (d) was taken by M.F-M.
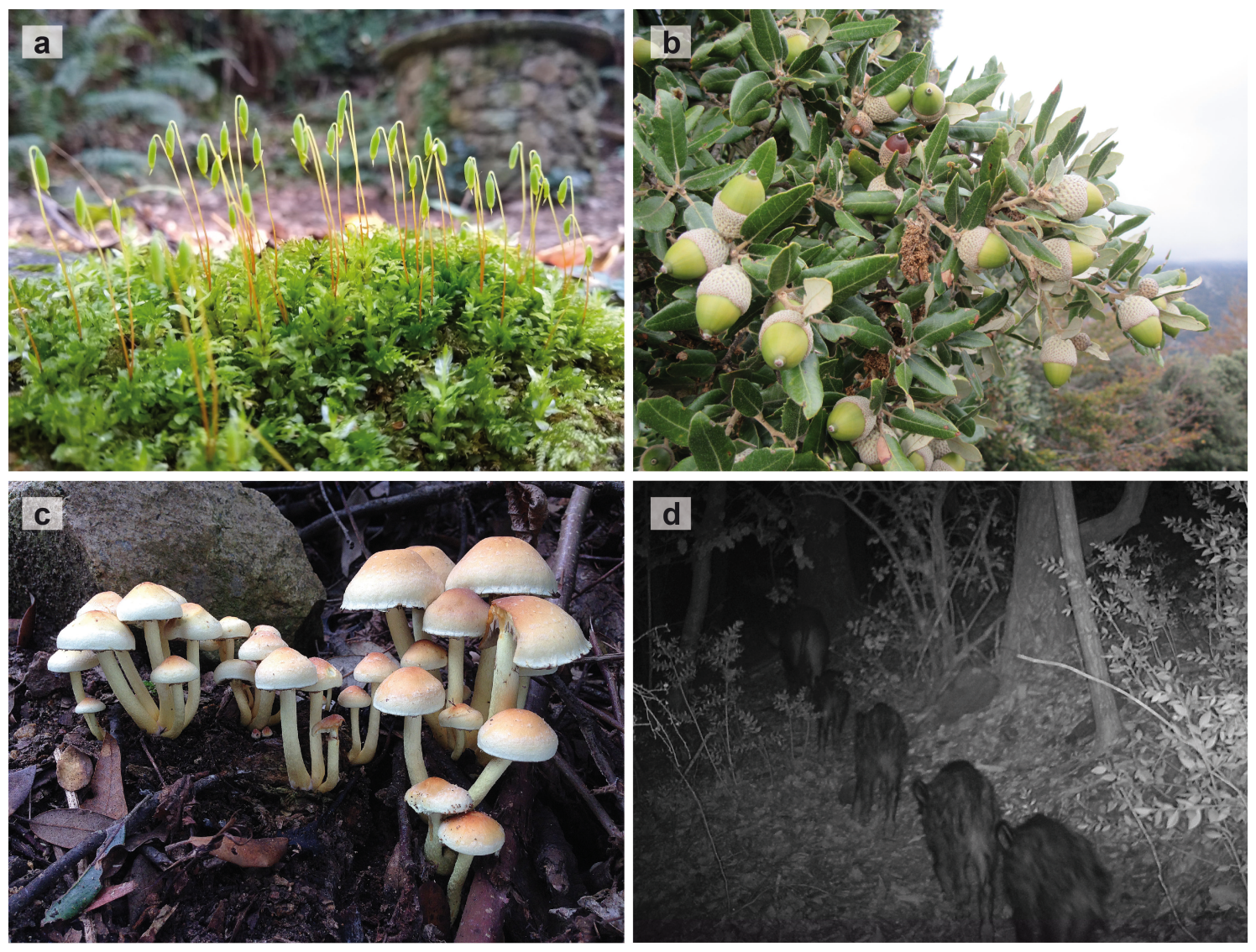